\title{
PILONIDAL DISEASE MIMICKING AS FISTULA-IN ANO - A RARE CASE REPORT
}

Pradeep Kumar. S, G. G. Betigeri, Safarulla Hasanrabba

1. Assistant Professor. Department of General Surgery, Dr. B. R. Ambedkar Medical College. Bangalore.

2. Associate Professor. Department of General Surgery, Dr. B. R. Ambedkar Medical College. Bangalore.

3. Resident. Department of General Surgery, Dr. B. R. Ambedkar Medical College. Bangalore.

\section{CORRESPONDING AUTHOR:}

Dr. Pradeep Kumar. S, No.108, Shivashakti Nilaya, $5^{\text {th }}$ cross, 11 th main, B.T.S. Layout, Siddedahalli, Bangalore- 560073.

E-mail: dr.pradeep_k20@yahoo.com

ABSTRACT: INTRODUCTION: Pilonidal disease (cyst, abscess and sinus) typically occur in the midline of the sacrococcygeal skin of young men. Pilonidal disease has been described in other parts of the body, such as the hands, umbilicus, axillae, and external genitalia. The following paper describes an unusual presentation of a pilonidal cyst with perianal drainage. Four cases were initially reported in 1948. A literature review found an additional 19 reported cases of pilonidal disease associated with a perianal fistula. PRESENTATION OF CASE: A 56- year- old male came with a chief complaint of peri-anal drainage for 3 months. He had persistent irritation associated with intermittent discharge and pain from his peri-anal region. Physical examination revealed a tender external perianal opening with a small amount of purulent drainage. A transrectal ultrasound revealed a sinus tract without any internal anal communication DISCUSSION: Chronic pilonidal disease is associated with sinus tract formation which is almost exclusively limited to the sacrococcygeal region but pilonidal disease has been described in other parts of the body, such as the hands, umbilicus, axillae, and external genitalia. The following paper describes an unusual presentation of a pilonidal cyst with perianal drainage. CONCLUSION: Although Pilonidal sinus usually occurs in sacrococcygeal region, the other sites should also be kept in mind and whenever internal opening of fistula-in-ano is not easily evident, it is better to get MRI or TRUS to make a definitive diagnosis as the two conditions can mimic each other.

KEY WORDS: Pilonidal, sinus, perianal, fistula-in-ano, hair, disease

INTRODUCTION: Pilonidal disease was first described by O . H . Mayo in 1833 although the term pilonidal was coined later by R. M . Hodges in 1880 [3]. The peak incidence is between 15 and 24 years of age. Other factors affecting its incidence are obesity, poor personal hygiene, increased sweating activity, and local trauma[2 ]. An acute abscess is the presenting feature in approximately $50 \%$ of patients with pilonidal disease [4,5]. Patients may present to the surgeon with findings of asymptomatic small midline pits in the gluteal cleft (which may contain hair) or as an obvious, painful abscess. After surgical or spontaneous drainage, chronic pilonidal disease may result [2].

The following paper represents an unusual presentation of pilonidal disease with perineal drainage. 
CASE REPORT: A 56- year- old male came with a chief complaint of peri-anal drainage for 3 months. He had persistent irritation associated with intermittent discharge and pain from his peri-anal region. Physical examination revealed a tender external perianal opening with a small amount of purulent drainage . A transrectal ultrasound revealed a sinus tract without any internal anal communication (Fig-2). It was decided to perform an examination under anesthesia and proceed.

On examination under anesthesia he had an external perianal opening at the 10 o'clock position , $4 \mathrm{~cm}$ from the anal verge with a small amount of mucous drainage (Fig-1). The external opening also revealed multiple hair follicles within the lumen of sinus. With a possible differential diagnosis of pilonidal sinus further examination was undertaken. On anoscopy, there was no obvious internal opening. The external opening was injected with hydrogen peroxide with methylene blue to identify any communication. On injection, blue dye or peroxide was not observed within the rectum. A fistula probe was then placed across the fistula tract however it ended blinded without any communication to anus. A large amount of hair and granulation tissue was debrided and the pilonidal cavity was excised entirely (Fig-3). The fistula tract was debrided and cavity was packed with Betadine soaked gauze and sterile bandages applied.

Postoperative wound healed well. At 1 month follow-up his wounds had granulated to the skin surface only to be covered with skin.

DISCUSSION: The patient with a pilonidal abscess typically presents with a history of increasing pain and the eventual development of a tender fluctuant mass in the sacrococcygeal area, often situated slightly off the midline. Cellulitis of the surrounding skin, as well as fever and leukocytosis, is occasionally present [2]. An acute abscess is the presenting finding in approximately $50 \%$ of patients with pilonidal disease $[4,5]$.Most patients with chronic pilonidal disease have pain, intermittent discharge, or both. They may present with recurrent bouts of infection. Rarely, they present with fever and chills. On physical examination, there may be evidence of past drainage with or without cellulitis and induration. The midline pit or pits are usually present, and hair may be protruding from the orifice. The differential diagnosis of sacrococcygeal pilonidal disease includes furuncle, hidradenitis suppurativa, fistula-in-ano, perianal abscess, sacral osteomyelitis with draining sinus, tuberculosis, and actinomycosis.[2]

Various treatment strategies for managing pilonidal disease have been employed. Most of these strategies focus on keeping the incision off the midline (where the wound is at risk for breakdown) and creating a shallower cleft. It is recommended to initially approach pilonidal disease with less-extensive procedures, reserving more complicated approaches for patients who develop chronic pilonidal disease. Patients who present acutely with an abscess are often treated with an elliptical incision made lateral to the midline which incorporates all of the diseased tissue (including sinus tracts) and allows healing by secondary intention. The recurrence rate is $20 \%$ in this setting [6].

Chronic pilonidal disease is associated with sinus tract formation which is almost exclusively limited to the sacrococcygeal region. The principals of management in this setting require excision of the sinuses and associated tracts. This can be done through incision and curettage of the tracts with or without marsupialization with recurrence rates of up to $19 \%$ [6]. This approach requires local wound care as the wound heals by secondary intention. Wide local excision with primary closure is usually associated with more wound complications and reported recurrence rates of $11-29 \%$ [6]. This dissection is taken down to the sacral fascia 
which likely contributes to the high incidence of wound complications by creating (or preserving) a deep cleft. Karydakis modified radical excision by proposing a curvilinear incision lateral to the midline with the creation of a gluteal flap and debridement of the sinus tracts [7]. This allows creation of a shallower cleft and keeps the wound off of the midline. Reported recurrence rates with this technique are less than 5\% [9]. More extensive reconstruction procedures have been described as well including the rhomboid flap , V- Y flaps, and Z -plasty [10]. These techniques are typically reserved for patients with multiple recurrences or nonhealing wounds. Due to the extensive tissue mobilization, when these techniques fail they can result in large wounds. Recurrence rates are less than $5 \%$ when successful [10] . A recent Cochrane database analysis of the surgical management of pilonidal disease concluded: "It may well be that off-midline closure is superior, but given the impression of the results and the lack of similarity across trials for some of the outcomes, better evidence is needed before suggesting it become standard of care." [11].

Pilonidal disease with fistula and drainage around the anus represents an atypical presentation that is more likely to raise a suspicion of fistula-in-ano rather than pilonidal disease. Four cases were initially reported in 1948 [12]. A literature review found an additional 19 reported cases of pilonidal disease associated with a perianal fistula ; however, we suspect that it is more prevalent than our literature search would reflect [13]. Primary perianal pilonidal disease is quite rare and can be treated with local excision with or without primary closure [13].

Pilonidal disease affecting a variety of other sites has also been reported including the perineum, interdigital spaces (i.e. , hairdressers, suggesting an acquired etiology ), umbilical , peritoneal catheter exit site, scalp, lateral buttock, and male and female genital tracts . Pilonidal disease itself has an incidence of approximately 1 in 4,000 [14].

In this subset of patients, the same principles for treating pilonidal disease must be employed in addition to obliteration of the fistula tract and preservation of the anal sphincter (although involvement of the sphincter by the fistula tract appears to be uncommon). Patients presenting with a perianal fistula should undergo a complete examination to identify an abscess, fistula-in-ano, or pilonidal disease. This can be achieved by an examination under anesthesia or the use of magnetic resonance imaging (MRI); the latter has become a useful adjunct in defining complex perianal disease and fistulous communications [3]. ); recently Transrectal Ultrasound (TRUS) has also been used with success which is cheaper compared to MRI but is operator dependent. The largest series of pilonidal disease with perianal fistula in the literature described using fistulectomy in combination with a Karydakis flap and reported no recurrences with that approach as well [13].

CONCLUSION: Although Pilonidal sinus usually occurs in sacrococcygeal region, the other sites should also be kept in mind and whenever internal opening of fistula-in-ano is not easily evident, it is better to get MRI or TRUS to make a definitive diagnosis as the two conditions can mimic each other.

CONSENT: Written informed consent was obtained from the patient for publication of this case report and accompanying images.

\section{REFERENCES:}


1. Sabiston Textbook of Surgery, 18th ed.

2. Shackelford's Surgery of the Alimentary Tract, sixth Edition

3. Taylor S A , Halligan S, Bartram C I. Pilonidal sinus disease: M R imaging distinction from fistula in ano. Radiology. 2003; 226(3): 662-667. [PubMed]

4. Da Silva J H. Pilonidal cyst: cause and treatment. Diseases of the Colon and Rectum. 2000; 43(8): 1146-1156. [PubMed

5. SMITH T E. Anterior or perineal pilonidal cysts . The Journal of the American Medical Association. 1948; 136(15): 973-975.

6. Sohn N , Martz J . Pilonidal disease . In : Cameron J L, editor. Current Surgical Therapy. 8 th edition. Philadelphia, Pa, USA: Elsevier, Mosby; 2004. pp. 280-284.

7. Karydakis G E. New approach to the problem of pilonidal sinus. The Lancet. 1973; 2 (7843): 1414-1415.

8. Bascom J. Surgical treatment of pilonidal disease. British Medical Journal. 2008; 336 (7649): 842-843. [PMC free article ] [PubMed]

9. Karydakis G E. New approach to the problem of pilonidal sinus. The Lancet. 1973; 2 (7843): 1414-1415.

10. Horwood J , Hanratty D, Chandran P, Billings P. Primary closure or rhomboid excision and Limberg flap for the management of primary sacrococcygeal pilonidal disease ? A meta analysis of randomized controlled trials. Colorectal Disease. 2012; 14(2): 143-151. [PubMed]

11. Brasel K J , Gottesman L, Vasilevsky C A, et al. Meta-analysis comparing healing by primary closure and open healing after surgery for pilonidal sinus . Journal of the American College of Surgeons. 2010; 211(3): 431-434. [PubMed]

12. SMITH T E. Anterior or perineal pilonidal cysts. The Journal of the American Medical Association. 1948; 136(15): 973-975.

13. Vallance S. Pilonidal fistulas mimicking fistulas-in-ano. British Journal of Surgery. 1982; 69(3): 161-162. [PubMed]

14. Bascom J. Surgical treatment of pilonidal disease . British Medical Journal . 2008; 336 (7649): 842-843. [PMC free article] [PubMed] 


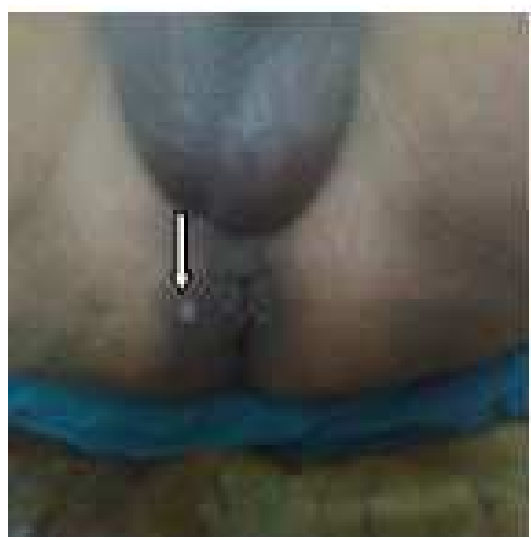

Fig-1: Pilonidal sinus in the perianal region mimicking as peri-anal fistula
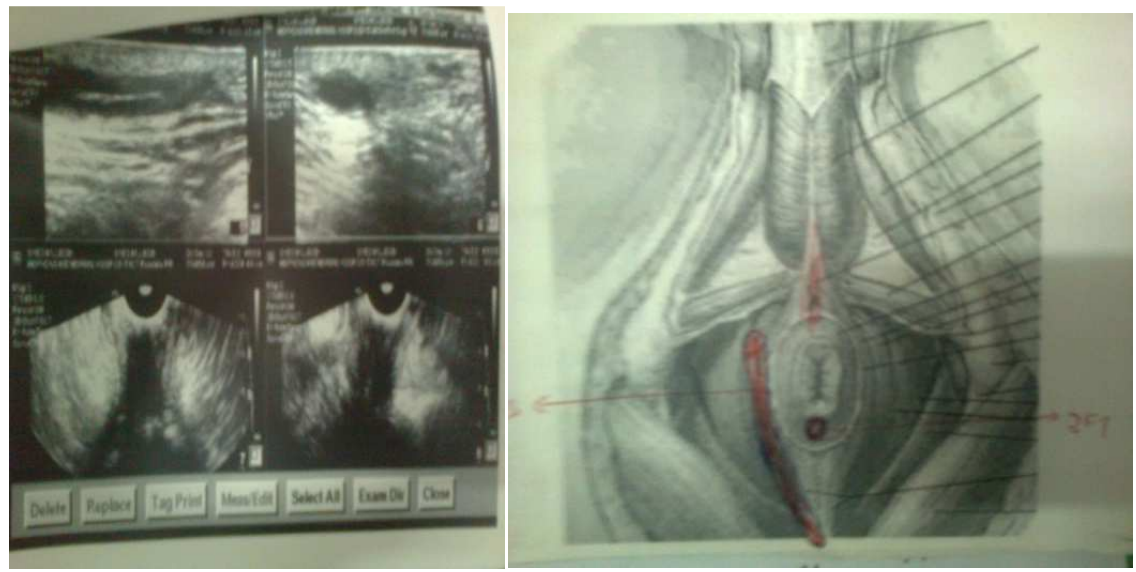

Fig-2: Transrectal ultrasound revealed a sinus tract without any internal anal communication

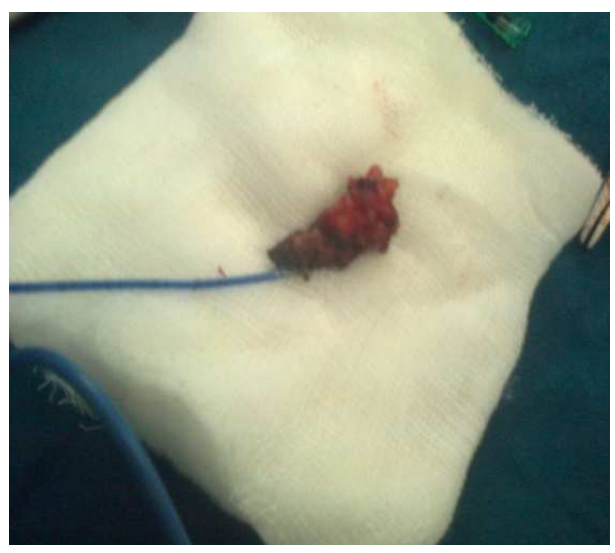

Fig-3 Excised pilonidal

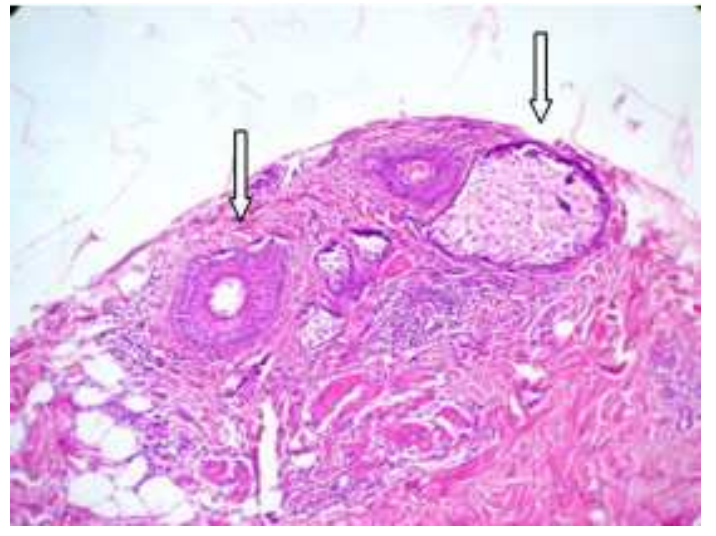

Fig 4:Histopathology slide showing granulation tissue covered by skin. Also seen are foci of tuft of hair deep in subcutaneous tissue suggestive of Sebaceous gland central hair canal with outer Root sheat. 